\title{
Low- and high-thermogenic brown adipocyte subpopulations coexist in murine adipose tissue
}

\author{
Anying Song, ${ }^{1}$ Wenting Dai, ${ }^{1}$ Min Jee Jang, ${ }^{2}$ Leonard Medrano, ${ }^{3}$ Zhuo Li, ${ }^{4}$ Hu Zhao, ${ }^{5}$ Mengle Shao, ${ }^{6}$ Jiayi Tan, ${ }^{1}$ Aimin Li, ${ }^{7}$ \\ Tinglu Ning, ${ }^{1}$ Marcia M. Miller, ${ }^{4}$ Brian Armstrong, ${ }^{8}$ Janice M. Huss, ${ }^{1}$ Yi Zhu, ${ }^{9}$ Yong Liu, ${ }^{10}$ Viviana Gradinaru, ${ }^{2}$ Xiwei Wu, ${ }^{11}$ \\ Lei Jiang, ${ }^{1,2}$ Philipp E. Scherer, ${ }^{6}$ and Qiong A. Wang ${ }^{1,12}$
}

\begin{abstract}
'Department of Molecular \& Cellular Endocrinology, Diabetes \& Metabolism Research Institute, City of Hope Medical Center, Duarte, California, USA. ²Division of Biology and Biological Engineering, California Institute of Technology, Pasadena, California, USA. ${ }^{3}$ Department of Translational Research \& Cellular Therapeutics, Diabetes \& Metabolism Research Institute, and ${ }^{4}$ Electron Microscopy and Atomic Force Microscopy Core, Beckman Research Institute, City of Hope Medical Center, Duarte, California, USA. 'Department of Restorative Sciences, School of Dentistry, Texas A\&M University, Dallas, Texas, USA. ${ }^{6}$ Touchstone Diabetes Center, University of Texas Southwestern Medical Center, Dallas, Texas, USA. ${ }^{7}$ Pathology Core of Shared Resources and ${ }^{8}$ Light Microscopy Digital Imaging Core, Beckman Research Institute, City of Hope Medical Center, Duarte, California, USA. ${ }^{9}$ Children's Nutrition Research Center, Department of Pediatrics, Baylor College of Medicine, Houston, Texas, USA. ${ }^{10} \mathrm{Hubei}$ Key Laboratory of Cell Homeostasis, College of Life Sciences, Institute for Advanced Studies, Wuhan University, Wuhan, China. "Integrative Genomics Core and ${ }^{12}$ Comprehensive Cancer Center, Beckman Research Institute, City of Hope Medical Center, Duarte, California, USA.
\end{abstract}

Brown adipose tissue (BAT), as the main site of adaptive thermogenesis, exerts beneficial metabolic effects on obesity and insulin resistance. BAT has been previously assumed to contain a homogeneous population of brown adipocytes. Utilizing multiple mouse models capable of genetically labeling different cellular populations, as well as single-cell RNA sequencing and 3D tissue profiling, we discovered a brown adipocyte subpopulation with low thermogenic activity coexisting with the classical high-thermogenic brown adipocytes within the BAT. Compared with the high-thermogenic brown adipocytes, these low-thermogenic brown adipocytes had substantially lower Ucp1 and Adipoq expression, larger lipid droplets, and lower mitochondrial content. Functional analyses showed that, unlike the high-thermogenic brown adipocytes, the lowthermogenic brown adipocytes have markedly lower basal mitochondrial respiration, and they are specialized in fatty acid uptake. Upon changes in environmental temperature, the 2 brown adipocyte subpopulations underwent dynamic interconversions. Cold exposure converted low-thermogenic brown adipocytes into high-thermogenic cells. A thermoneutral environment had the opposite effect. The recruitment of high-thermogenic brown adipocytes by cold stimulation is not affected by high-fat diet feeding, but it does substantially decline with age. Our results revealed a high degree of functional heterogeneity of brown adipocytes.

\section{Introduction}

Brown adipose tissue (BAT) is a thermogenic organ that is thought to play an important role in human energy homeostasis (1-3). Upon activation, brown adipocytes within the BAT can function as an effective energy sink, burning and disposing excess lipids and glucose (4-6). In recent years, progress has been made in rodents and humans in understanding the function and physiological impact of BAT. It is now well accepted that recruiting and activating BAT can correct dyslipidemia and prevent obesityrelated metabolic disorders (7-10). Although functional heterogeneity has recently been reported in white and beige adipocytes within an individual fat depot (11-14), BAT is still viewed to have a highly homogeneous population of brown adipocytes. Interest-

\section{Related Commentary: p. 65}

Conflict of interest: The authors have declared that no conflict of interest exists. Copyright: ( 2020, American Society for Clinical Investigation. Submitted: March 29, 2019; Accepted: September 25, 2019; Published: November 25, 2019.

Reference information: J Clin Invest. 2020;130(1):247-257 https://doi.org/10.1172/JCI129167. ingly, some previous studies have indicated that thermogenesis is not uniformly activated in all brown adipocytes. For instance, brown adipocytes have been shown to have a heterogeneous expression of uncoupling protein 1 (UCP1) $(15,16)$. Moreover, in vitrocultured brown adipocytes showed heterogeneous mitochondrial membrane potential $(17,18)$. However, the thermogenic and metabolic heterogeneity of brown adipocytes within the same BAT in vivo remains largely uncharacterized.

\section{Results}

Brown adipocytes heterogeneously and dynamically express Adipoq. To better understand brown adipocyte dynamics in vivo, we used the AdipoChaser-LacZ mouse model we previously developed to label brown adipocytes. This model is a doxycycline-based (doxbased), tet-responsive labeling system for the inducible, permanent labeling of adiponectin-expressing (Adipoq-expressing) cells as LacZ ${ }^{+}$cells (Supplemental Figure 1A and refs. 19, 20; supplemental material available online with this article; https://doi. org/10.1172/JCI129167DS1). To our surprise, at room temperature $\left(24^{\circ} \mathrm{C}\right)$, despite the uniform labeling of white adipocytes (19, 20 ), only $38 \%$ of total brown adipocytes in the BAT were labeled as $\mathrm{LacZ}^{+}$(blue) cells, and these cells distribute in a patchy pattern 
A

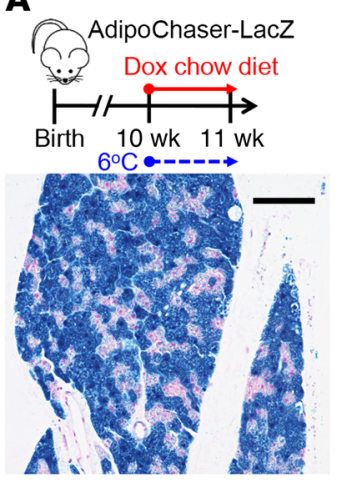

C

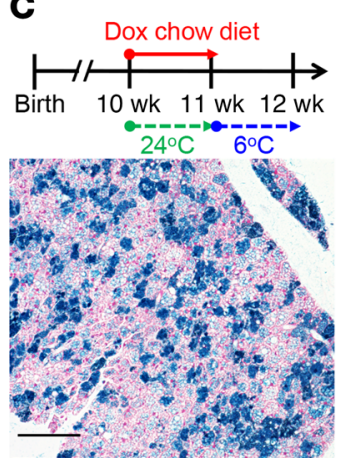

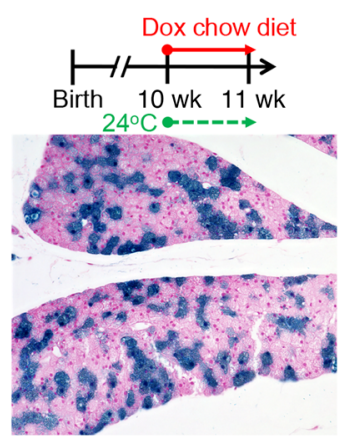

$D$

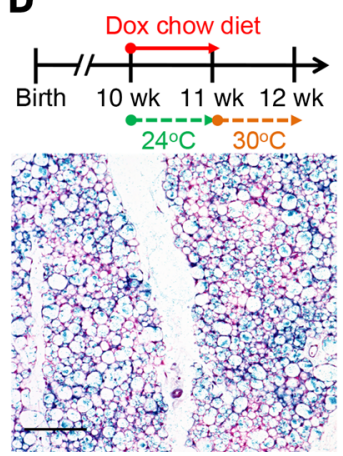

B
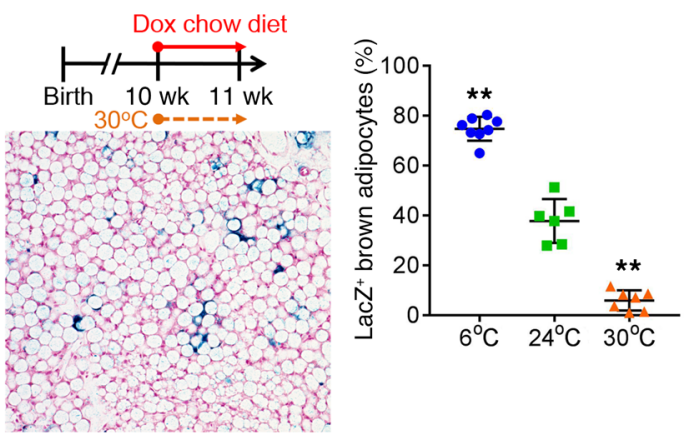

E

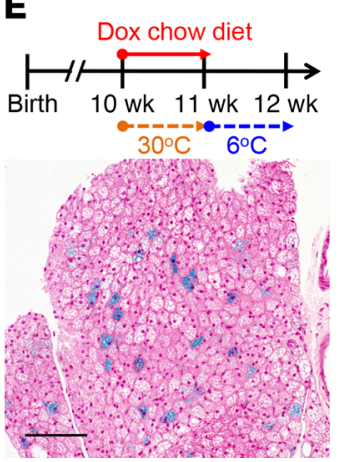

$\mathbf{F}$

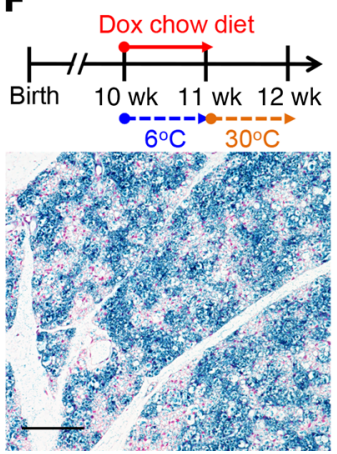

Figure 1. Two subpopulations of classical brown adipocytes undergo dynamic interconversions in vivo. (A) Representative X-gal staining of BAT from AdipoChaser-LacZ mice exposed to different environmental temperatures while fed with dox-containing chow diet. (B) Quantification of the percentage of Lac $Z^{+}$brown adipocytes in the total brown adipocytes. $n=8$ mice $\left(6^{\circ} \mathrm{C}\right) ; 6$ mice $\left(24^{\circ} \mathrm{C}\right) ; 7$ mice $\left(30^{\circ} \mathrm{C}\right)$. (C-F) Representative $\mathrm{X}$-gal staining of BAT from AdipoChaser-LacZ mice kept at the indicated temperatures while fed with dox-containing chow diet, followed by regular chow diet feeding at the indicated temperatures. Scale bars: $100 \mu \mathrm{m}(\mathbf{A}, \mathbf{C}-\mathbf{F})$. All data are mean \pm SD of biologically independent samples; ${ }^{* *} P<0.01$. Statistical significance was assessed using a 1-way ANOVA followed by Tukey's multiple comparisons test. All images are representative of 3 independent experiments.

(Figure 1, A and B). The percentage of $\mathrm{LacZ}^{+}$brown adipocytes was markedly higher $(76 \%)$ when mice were housed in a cold environment $\left(6^{\circ} \mathrm{C}\right)$ and markedly lower $(6 \%)$ when mice were housed in a thermoneutral environment $\left(30^{\circ} \mathrm{C}\right)$ (Figure $1, \mathrm{~A}$ and $\left.\mathrm{B}\right)$. However, Adipoq mRNA in the whole BAT was slightly increased when mice were at $6^{\circ} \mathrm{C}$, and was not altered when mice were in $30^{\circ} \mathrm{C}$ (Supplemental Figure 1B). When we treated AdipoChaser-LacZ mice with $\beta 3$-adrenergic receptor agonist to stimulate thermogenesis (Supplemental Figure 1C), we observed a similar percentage of $\mathrm{LacZ}^{+}$ brown adipocytes as was seen upon cold exposure (67\%) (Supplemental Figure 1, D and E).

Is the increase of $\mathrm{LacZ}^{+}$brown adipocytes during cold exposure due to de novo adipogenesis? And likewise, is the decrease of $\mathrm{LacZ}^{+}$brown adipocytes during thermoneutral exposure due to cell death? When we prelabeled mice at $24^{\circ} \mathrm{C}$ and pulse-chased at $6^{\circ} \mathrm{C}$ or $30^{\circ} \mathrm{C}$, the percentages of $\mathrm{LacZ}^{+}$brown adipocytes $(40 \%)$ remained the same as when they were at $24^{\circ} \mathrm{C}$ (Supplemental Figure $1, \mathrm{C}$ and D). When we prelabeled mice at $30^{\circ} \mathrm{C}$ and pulse-chased at $6^{\circ} \mathrm{C}$, the percentages of $\mathrm{LacZ}^{+}$brown adipocytes $(5 \%)$ remained the same as when they were at $30^{\circ} \mathrm{C}$ (Figure 1E). Likewise, when we prelabeled mice at $6^{\circ} \mathrm{C}$ and pulse-chased at $30^{\circ} \mathrm{C}$, the percentages of $\mathrm{LacZ}^{+}$brown adipocytes (73\%) remained the same as when they were at $6^{\circ} \mathrm{C}$ (Figure $\left.1 \mathrm{~F}\right)$. Meanwhile, body weight, BAT weight, and brown adipocyte cell size were not altered when mice were in a cold environment (Supplemental Figure 1, F-H). More- over, we have not seen obvious apoptosis of brown adipocyte by active caspase 3 staining (Supplemental Figure 2, A-D). Therefore, there are dynamic interconversions between these 2 brown adipocyte subpopulations upon temperature change, and we have no evidence of significant adipogenesis or cell death.

The Adipoq low-expressing brown adipocyte subpopulation has unique subcellular morphology and lower UCP1 expression. We subsequently looked into the subcellular structure of these 2 brown adipocyte subpopulations through electron microscopy imaging. $\mathrm{X}$-gal, when cleaved by $\beta$-galactosidase, produces 5,5'-dibromo-4,4'-dichloro-indigo-2, an intense blue product which is insoluble. Under the electron microscope, this blue product can be observed as crystals $(21,22)$, and the $\mathrm{LacZ}^{+}$brown adipocytes can be distinguished by this feature. Compared with the $\mathrm{LacZ}^{+}$brown adipocytes (Adipoq highexpressing), the LacZ- brown adipocytes had markedly lower mitochondrial number/content and much larger lipid droplets (Figure 2, A-D). We then switched to an AdipoChaser-mT/mG system we reported recently (refs. 20, 23 and Supplemental Figure $3 \mathrm{~A}$ ), and confirmed that Adipoq is selectively expressed in a subpopulation of brown adipocytes in a patchy pattern (Supplemental Figure 3, B and C). In the isolated primary brown adipocytes, the $\mathrm{GFP}^{-}$(Adipoq low-expressing) brown adipocytes had markedly higher mitochondrial membrane potential (Supplemental Figure 3, D-F), indicating that these cells have lower 
A

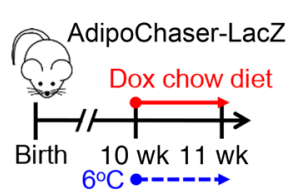

C

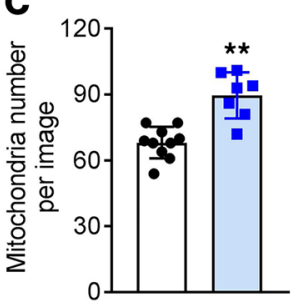

D

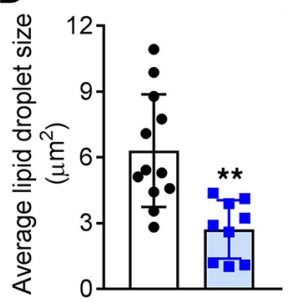

LacZ-

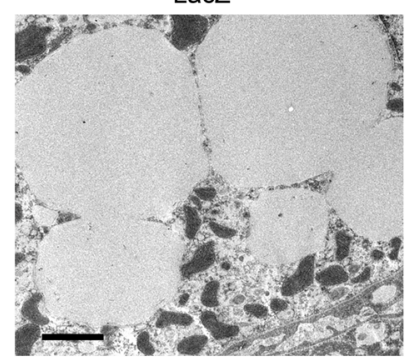

$\mathrm{LacZ}^{+}$

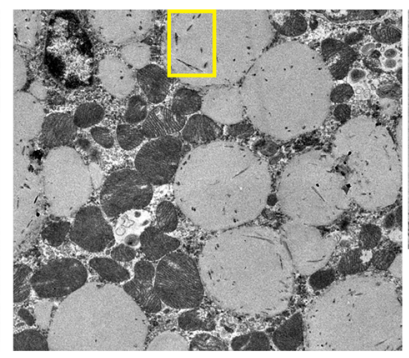

B

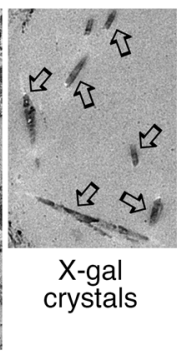

- LacZ- brown adipocyte

- LacZ+ brown adipocyte

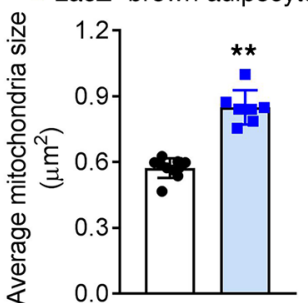

E
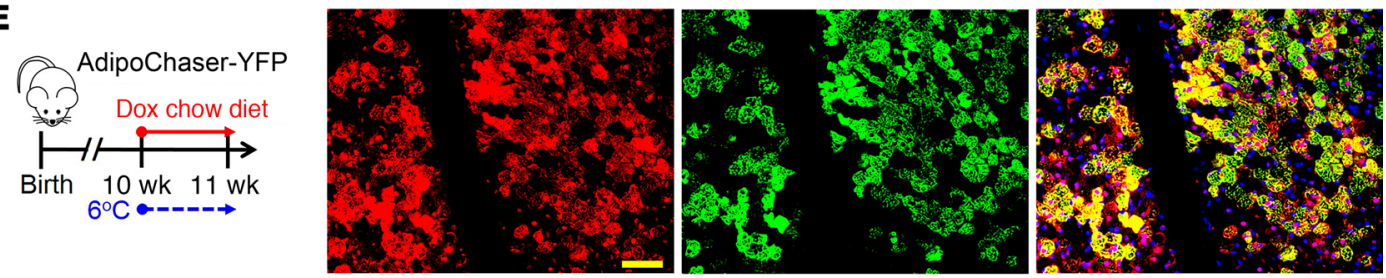

$\mathbf{F}$
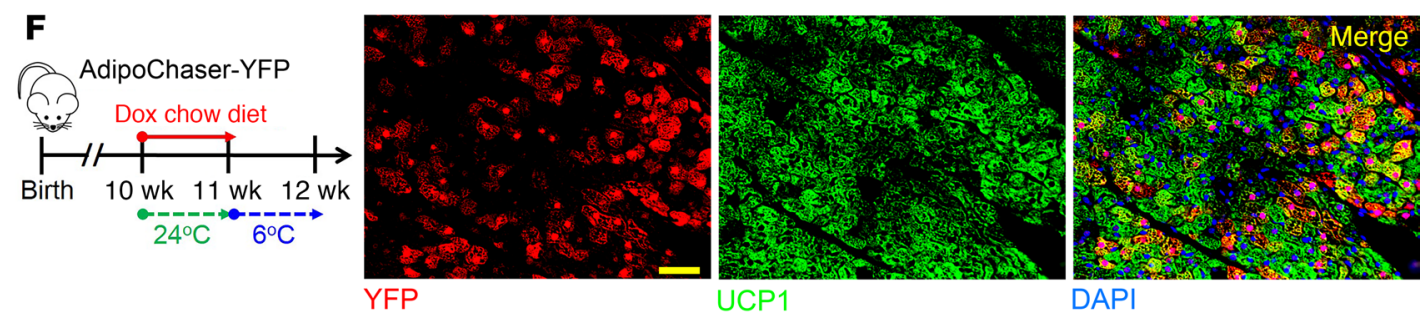

Figure 2. The 2 brown adipocyte subpopulations have distinct morphology and Ucp1 expression. (A) Electron micrographs of BAT from mice kept at $6^{\circ} \mathrm{C}$. Arrows: LacZ crystals. Scale bar: $2 \mu \mathrm{m}$. (B-D) Quantification of the mitochondria size, number, and lipid droplet size. $n=7$ LacZ ${ }^{+}$cells for $\mathbf{B}$ and $\mathbf{C}, n=9$ LacZ ${ }^{+}$cells for $\mathbf{D} ; n=10$ LacZ $^{-}$cells for $\mathbf{B}$ and $\mathbf{C}, n=12$ LacZ $^{-}$cells for $\mathbf{D}$. (E and $\mathbf{F}$ ) YFP (red), UCP1 (green), and DAPI (blue) immunofluorescence staining of BAT from AdipoChaser-YFP mice treated with dox-containing chow diet as indicated. Cells with yellow color are double positive with YFP and UCP1. Scale bars: $50 \mu \mathrm{m}$. All data represent mean \pm SD of biologically independent samples; ${ }^{* *} P<0.01$. Statistical significance was assessed using a 2-tailed Student's $t$ test (B-D). All images are representative of 3 independent experiments.

mitochondrial membrane depolarization and uncoupling rate (24). We also generated the AdipoChaser-YFP mice (Supplemental Figure $3 \mathrm{G}$ ), as YFP is relative easier for immunofluorescence staining. When we labeled mice in $6^{\circ} \mathrm{C}$, the Adipoq highexpressing $\left(\mathrm{YFP}^{+}\right)$brown adipocytes largely overlapped with UCP1 high-expressing cells (Figure 2E). Thus, Adipoq expression positively correlates with UCP1 protein expression. Overall, these results suggest that the Adipoq low-expressing brown adipocytes are morphologically and molecularly different from the Adipoq high-expressing brown adipocytes. When we prelabeled mice at $24^{\circ} \mathrm{C}$ and pulse-chased at $6^{\circ} \mathrm{C}$, there were markedly more $\mathrm{UCP}^{+}$cells than $\mathrm{YFP}^{+}$cells, and most of the prelabeled $\mathrm{YFP}^{+}$brown adipocytes colabeled as $\mathrm{UCP}^{+}$cells (Figure $2 \mathrm{~F}$ ). This result confirms that the YFP- brown adipocytes labeled at $24^{\circ} \mathrm{C}$ could convert into UCP1 high-expressing cells at $6^{\circ} \mathrm{C}$.

Molecular heterogeneity between the 2 brown adipocyte subpopulations revealed by single-cell RNA sequencing. We next set out to verify the brown adipocyte heterogeneity through single-cell RNA sequencing (scRNA-seq) of primary brown adipocytes isolated from the BAT of adult mice housed at $24^{\circ} \mathrm{C}$. Two major brown adipocyte subpopulations were clustered: brown adipocytes with high thermogenic activity (BA-H, 2352 cells, which includes 3 subclusters, BA-H1, BA-H2, and BA-H3) and brown adipocytes with low thermogenic activity (BA-L, 1250 cells) (Fig- ure 3A). These 2 populations differed by the expression level of Ucp1 as well as Adipoq (Figure 3, B and C). Two other clusters of cells were identified as white adipocytes (WA, 197 cells) and nonadipocytes (NA, 34 cells) (Figure 3A). The white adipocyte cluster served as an internal control in the subsequent analysis. In the BA-L subpopulation, expressions of genes related to thermogenesis, such as Cidea, Elovl6, and oxidative phosphorylation (OXPHOS) complexes, were extremely low, close, or lower than the white adipocytes in the WA cluster (Figure 3D, Supplemental Figure 4, and Supplemental Figure 5, A-E). Similarly, the BA-L subpopulation had very low expression levels of genes related to lipolysis, glycolysis, fatty acid oxidation, and the TCA cycle (Figure 3E and Supplemental Figure 6, A-C). Moreover, 2 newly identified pathways that have been described and may be essential for the positive regulation of thermogenesis, ROS (25) and succinate metabolism (26), were also only enriched in the BA-H subpopulation (Supplemental Figure 6, D and E). Therefore, brown adipocytes within the BA-L subpopulation belong to a novel and unique type of brown adipocyte with low thermogenic activity.

Notably, the BA-L subpopulation had substantial high expression levels of genes related to fatty acid uptake (Figure 3F and Supplemental Figure 7A). This subpopulation was also enriched for genes that are essential for cell-to-cell trafficking (ref. 27 and Supplemental Figure 7B), as well as UCP1-independent thermo- 

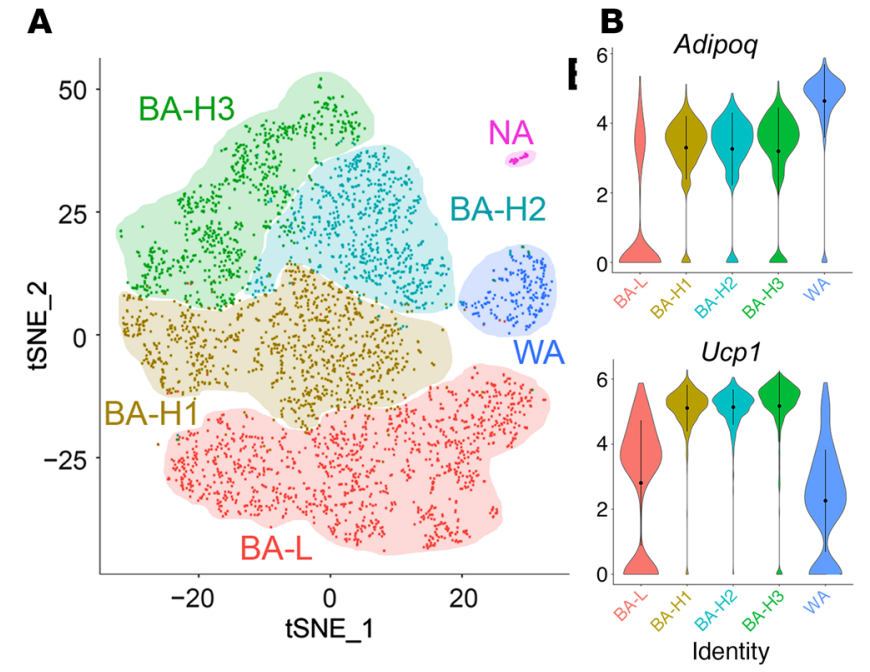

C
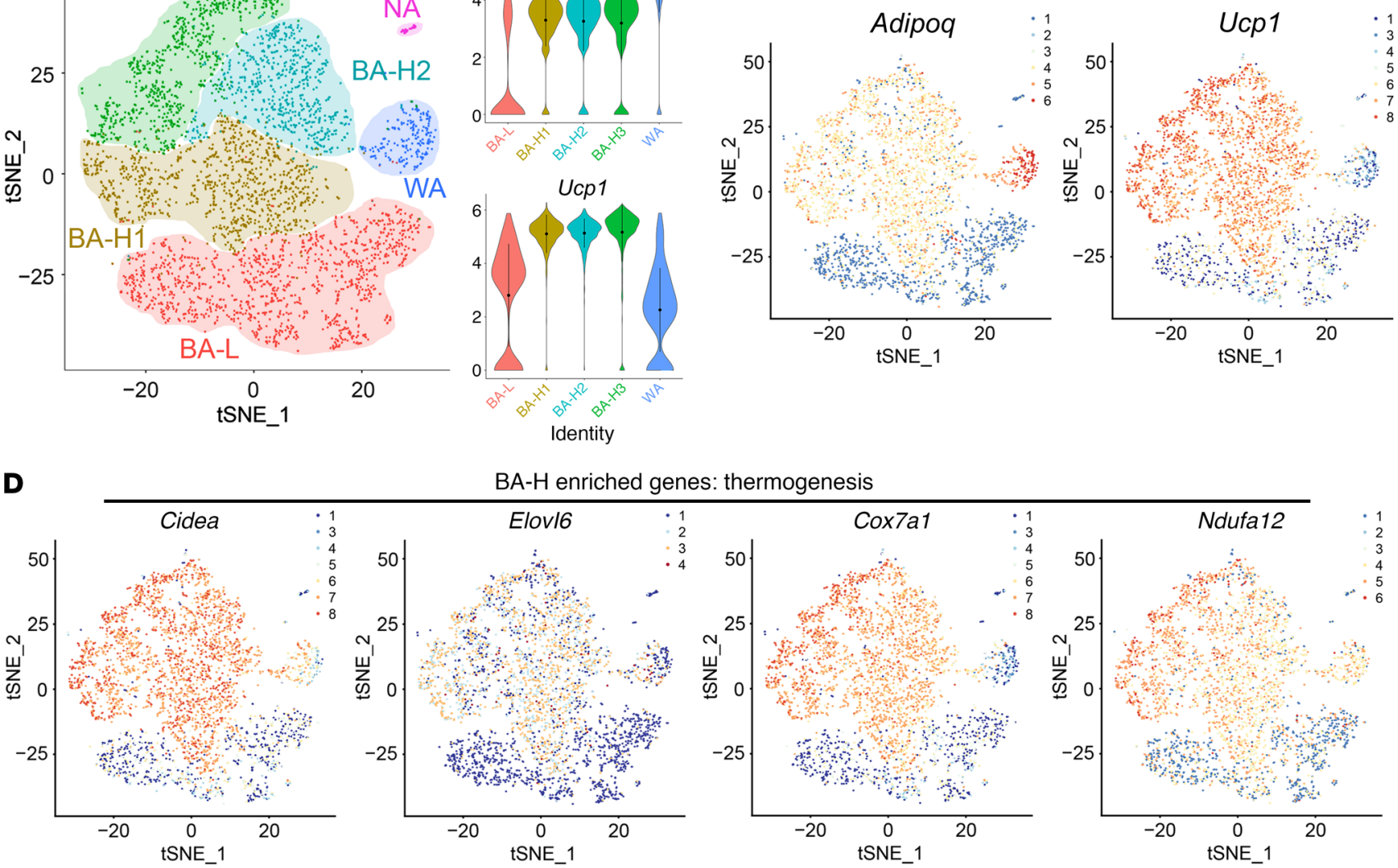

BA-H enriched genes: thermogenesis
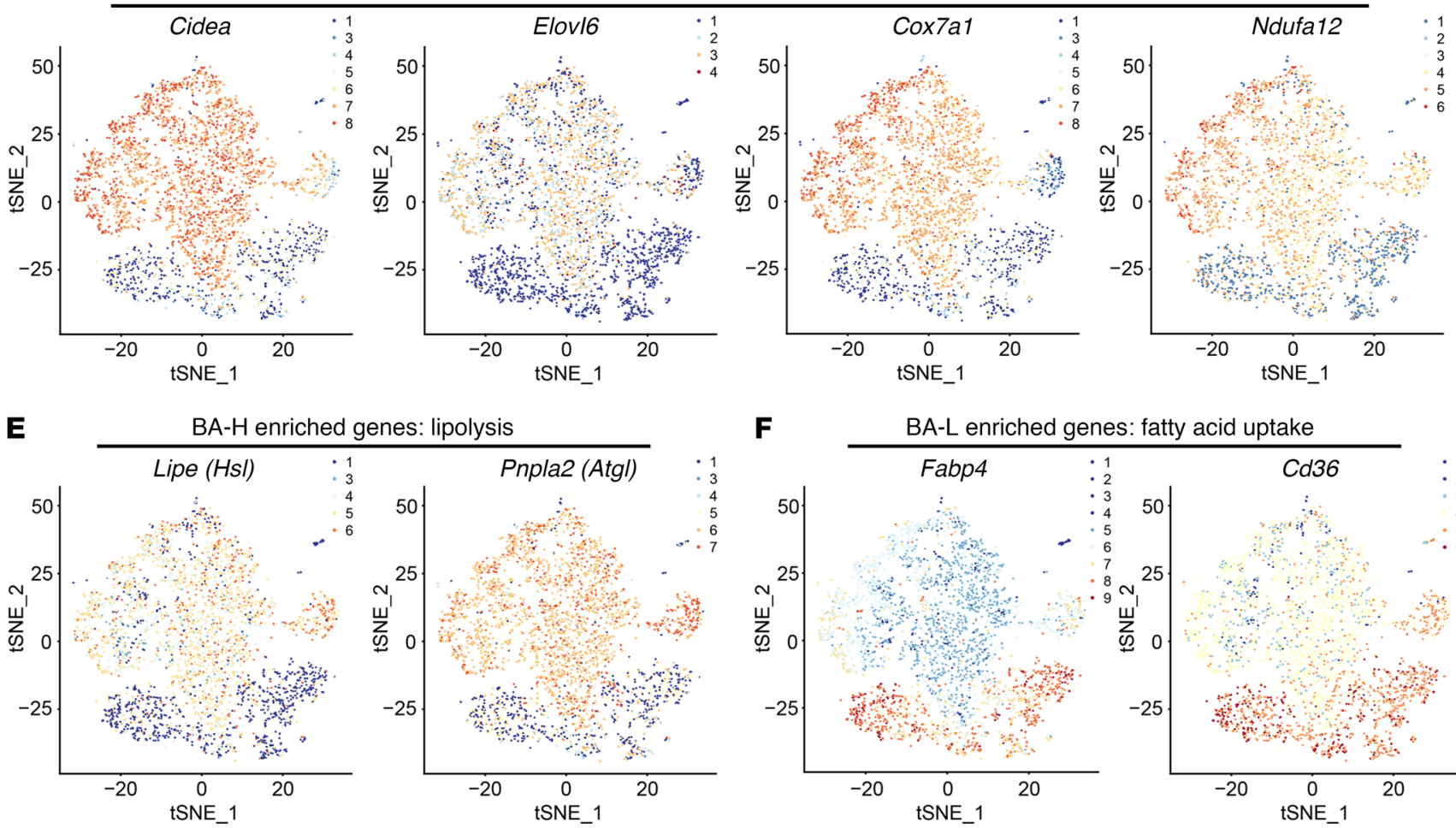

F $\quad$ BA-L enriched genes: fatty acid uptake
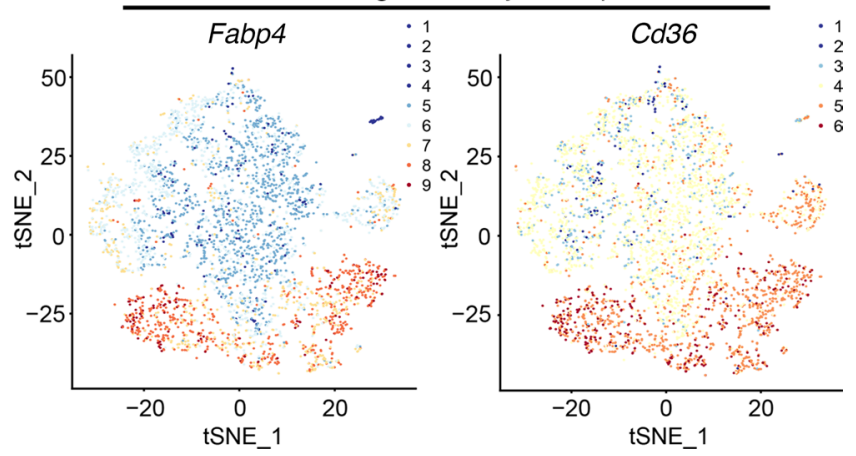

Figure 3. Single-cell RNA sequencing confirms the existence of $\mathbf{2}$ distinct brown adipocyte subpopulations. (A) t-distributed stochastic neighbor embedding (tSNE) plot of 3602 primary brown adipocytes isolated from 10-week-old WT male mice. Clustering was generated using $\mathrm{k}$-means $=4$. These data are from a single experiment. (B-F) Transcript counts represent $\log _{2}$ of gene expression. Each dot corresponds to one single cell, colored according to cell cluster. (B) Violin plots showing the distribution of normalized expression values of adiponectin (Adipoq) and Ucp1 across cells that belong to the 5 adipocyte clusters. (C-F) Distribution of the expression of Adipoq and Ucp1 (C), Cidea, Elovl6, Cox7a1, and Ndufa12 (D), Lipe (Hsl) and Pnpla2 (Atgl) (E), and Fabp4 and Cd36 (F) within tSNE plot.

genesis through the futile cycle of creatine metabolism (28) and tight junction (Supplemental Figure 7, C and D). Thus, brown adipocytes within the BA-L subpopulation hold a unique metabolic status, and the function of these cells is potentially fundamentally different from the cells within the BA-H subpopulation.

What regulates the functional heterogeneity between the 2 brown adipocyte subpopulations? Interestingly, PPAR $\gamma$ (29) and C/EBP $\alpha$ (30), the 2 master transcription factors that regulate adipocyte function $(31,32)$, have distinct expression patterns in these 2 subpopulations. Surprisingly, Pparg was relatively enriched in the BA-L subpopulation (Supplemental Figure 7E), consistent with the expression patterns of its downstream targets $\mathrm{Cd} 36$ and Fabp4 (Figure 3F). In contrast, Cebpa was enriched in the BA-H subpopulation (Supplemental Figure $7 \mathrm{E})$. These data indicate that these 2 factors may act as upstream regulators responsible for the distinct transcriptional profiles of the 2 brown adipocyte subpopulations. As expected, the expression of white adipocyte marker resistin (Retn) (Supplemental 
A

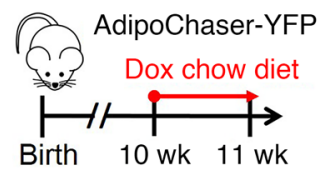

B

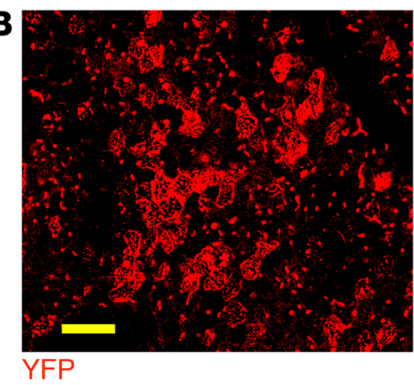

C

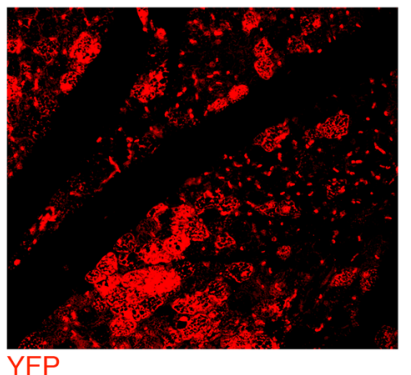

D

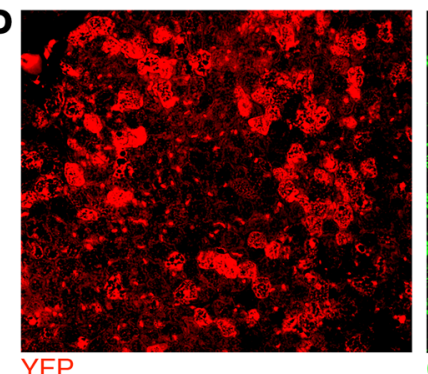

YFP

$\mathbf{E}$

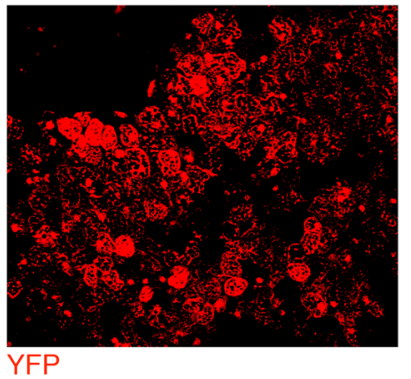

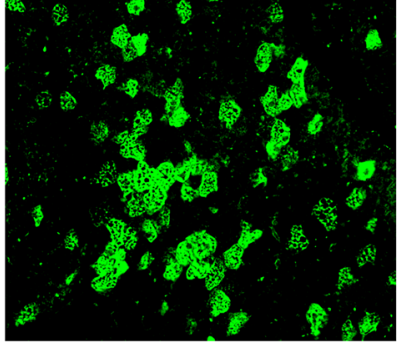

Elovl6

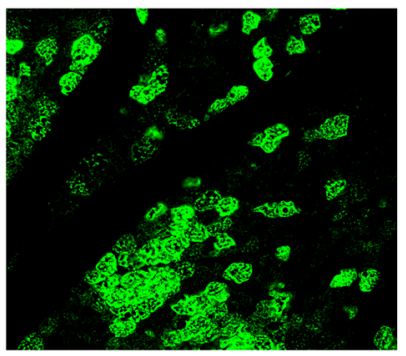

SDHA

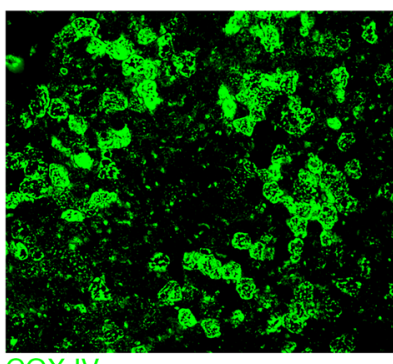

COX IV

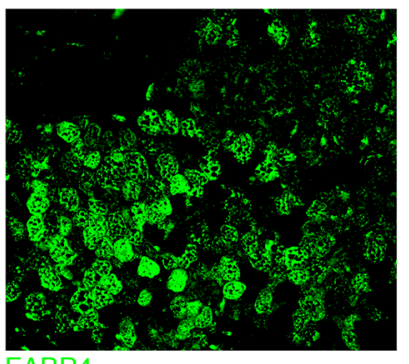

FABP4

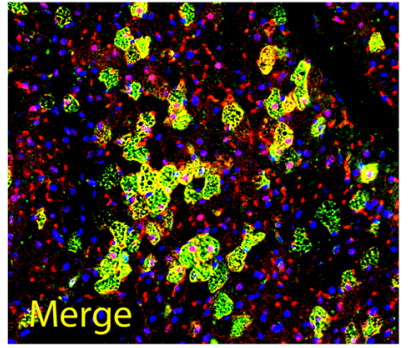

DAPI
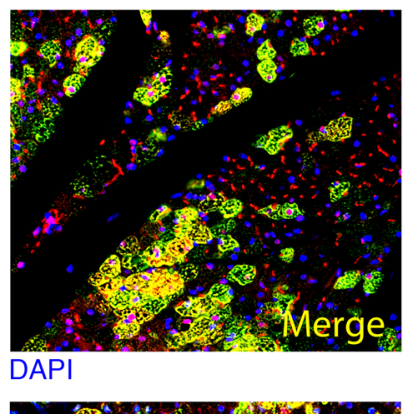

Figure 4. Immunofluorescence costaining of differentially expressed genes identified through single-cell RNA sequencing. (A) AdipoChaser-YFP male mice were kept on normal chow until 10 weeks of age. Mice were then treated with dox-containing chow diet for 1 week. (B-F) YFP (red) and Elovl6 (green) (B), SDHA (green) (C), COXIV (green) (D), FABP4 (green) (E), and PPAR $\gamma$ (green) (F) immunofluorescence staining of BAT from AdipoChaserYFP mice treated with dox-containing chow diet as indicated. Cells with yellow color are merged from red and green. Scale bars: 50 $\mu \mathrm{m}$. Images are representative of 2 independent experiments.

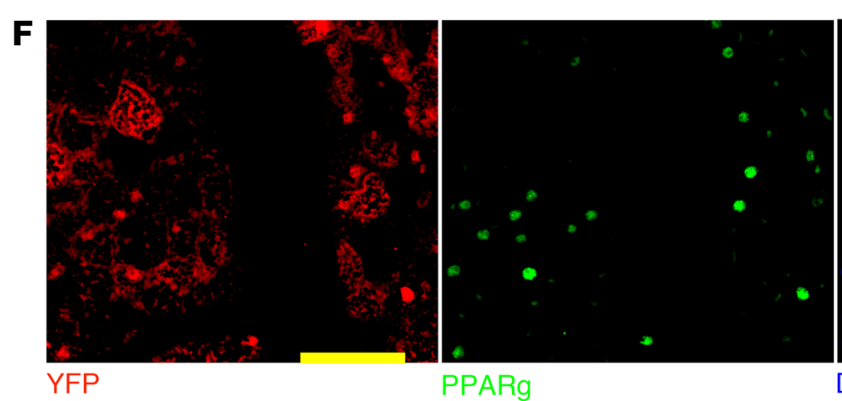

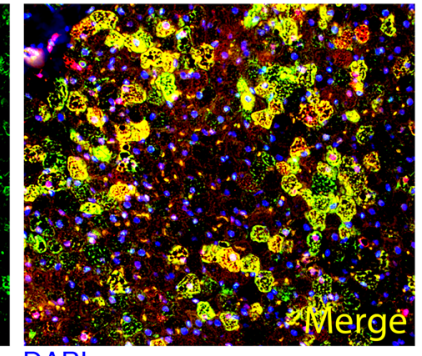

DAPI

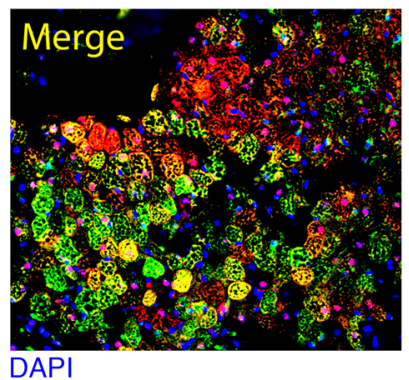

Figure 7F) was detected only in the WA cluster. Among the 3 BA-H subclusters, thermogenic genes had the highest expression levels in BA-H3 (Supplemental Figure 4). The subclusters $\mathrm{BA}-\mathrm{H} 1$ and $\mathrm{BA}-\mathrm{H} 2$ were enriched for genes that related to mito- chondrial biogenesis and insulin responsiveness (refs. 33-35 and Supplemental Figure $7 \mathrm{H}$ ).

We next performed immunofluorescence costaining to confirm the protein levels of these genes identified through scRNA- 
A

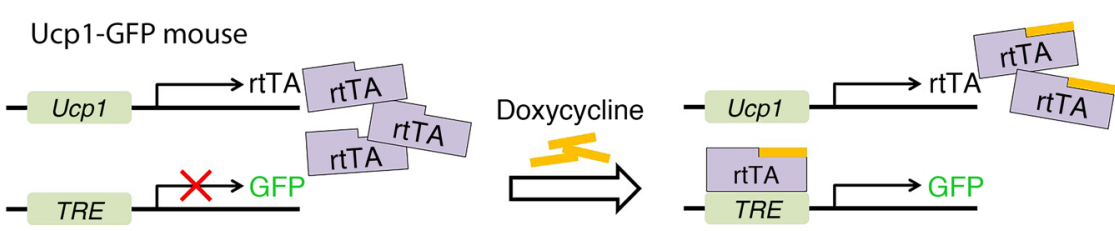

B

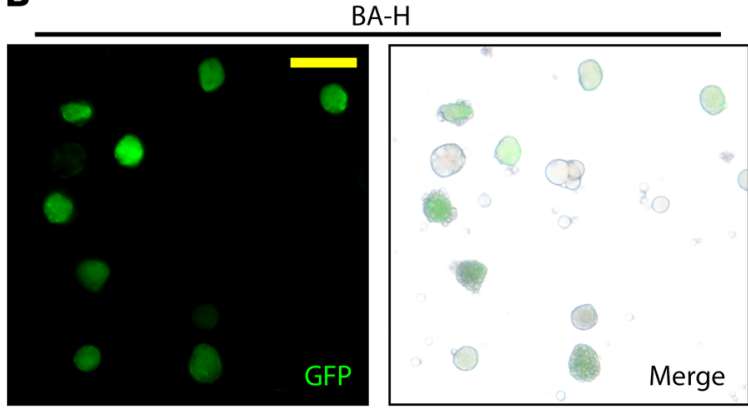

C

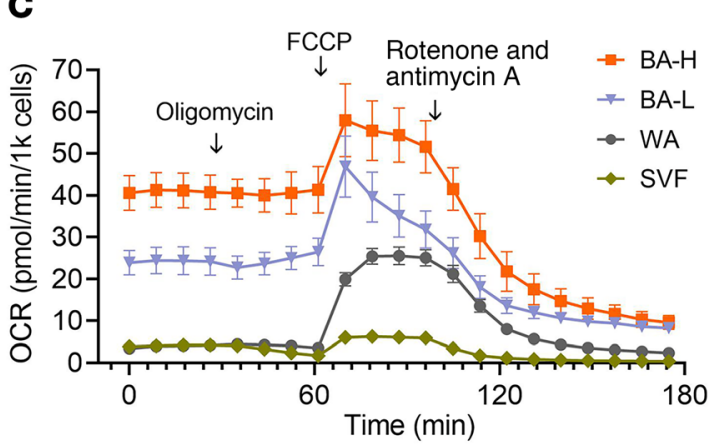

$\mathbf{F}$
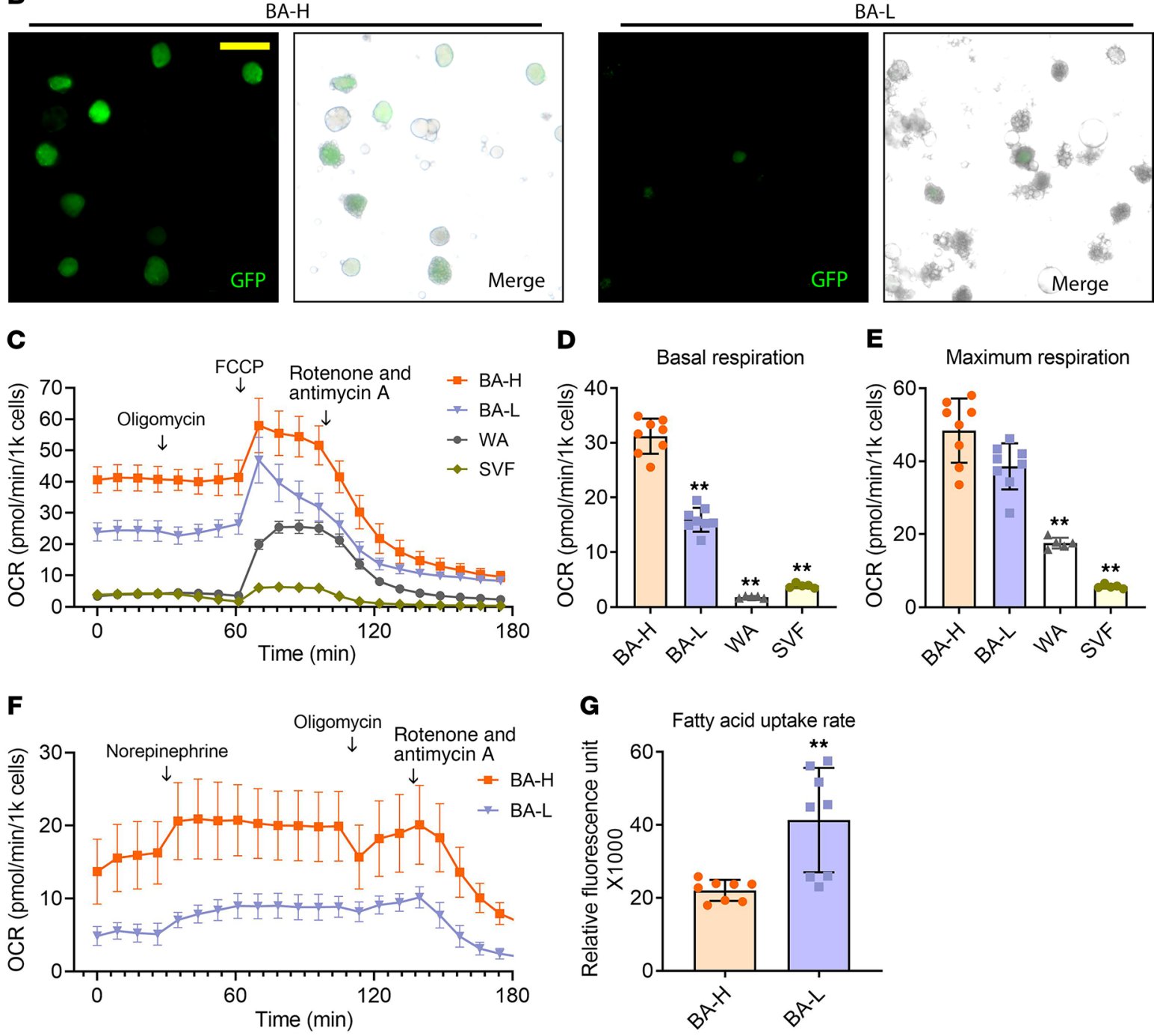

\section{E}
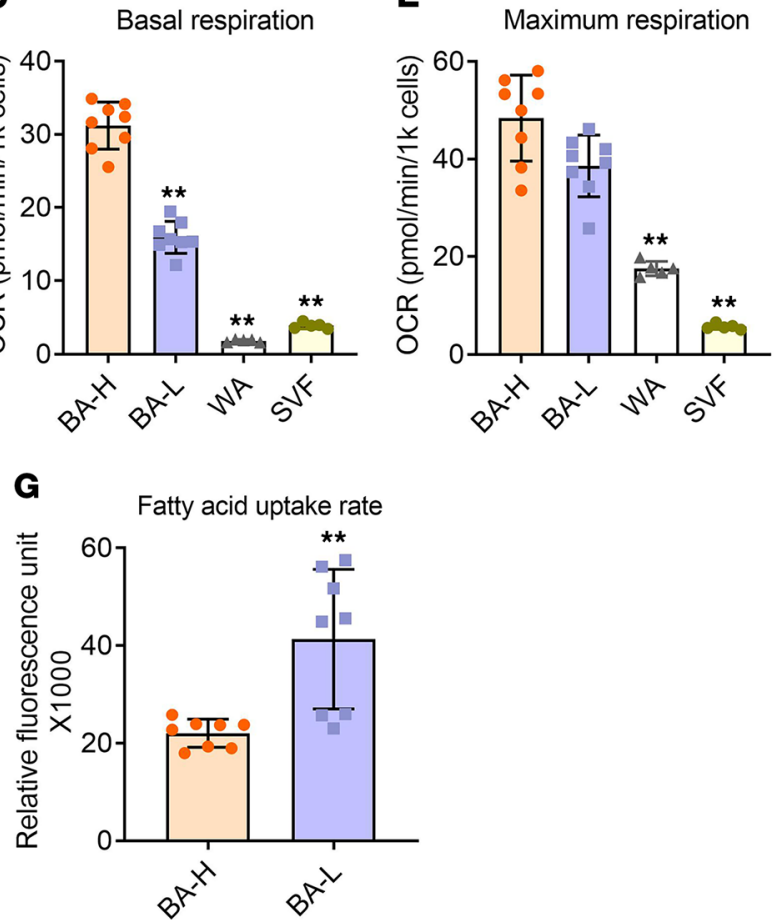

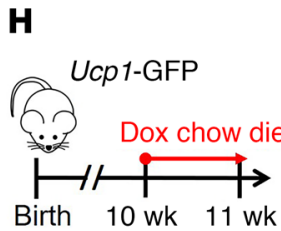

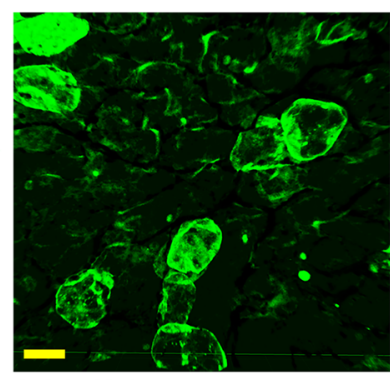

GFP (UCP1)

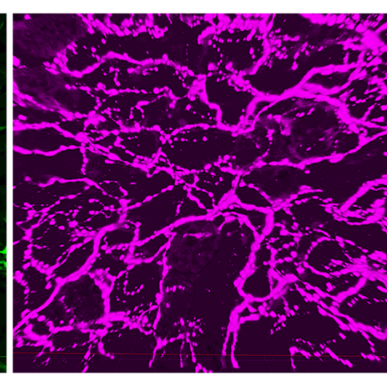

TH

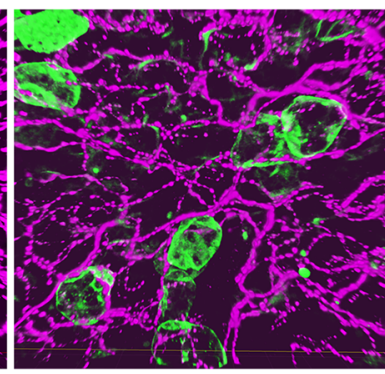

Merge 
Figure 5. The brown adipocyte subpopulations have distinct metabolic profiles. (A) Ucp1-GFP mice, the inducible real-time labeling system of Ucp1 promoter activity, derived from interbreeding 2 transgenic strains, Ucp1-rtTA and TRE-GFP, which allows inducible real-time labeling of the Ucp1 promoter activity. At the basal level, Ucp1-GFP mice do not express GFP in any cell type. When these mice are treated with dox, GFP expression is induced based on the Ucp1 promoter activity. (B) Fluorescent images of isolated BA-H and BA-L subpopulations from the BAT of Ucp1-GFP mice. Scale bar: $50 \mu \mathrm{m}$. These images are representative of 4 independent experiments. (C-E) OCR in freshly isolated primary cells treated with the different compounds. Primary brown adipocytes (BA-H and BA-L) were from male WT mice housed in $6^{\circ} \mathrm{C}$ for 7 days. As controls, primary white adipocytes and SVF from BAT were from male WT mice housed at room temperature. (C) Plot of OCR to time measured by Seahorse. (D and E) Calculated basal and maximum respiration levels of different cell types. $n=8$ mice (BA-H and BA-L); $n=5$ mice (WA and SVF). (F) Plot of OCR to time measured by Seahorse in primary brown adipocytes (BA-H and BA-L) treated with the different compounds. These cells were freshly isolated from male AdipoChaser-mT/mG mice housed in $6^{\circ} \mathrm{C}$ for 7 days through EasySep Magnet (Stem Cell Technologies). (C) Fatty acid uptake rate in the 2 brown adipocyte subpopulations. $n=8$ mice (BA-H and BA-L). For each group, cells from all mice were pooled together, and data represent mean $\pm \mathrm{SD}$ of experimental replicates, normalized to cell numbers. ${ }^{*} P<0.01$. Statistical significance was assessed using a 1-way ANOVA followed by Tukey's multiple comparisons test ( $\mathbf{D}$ and $\mathbf{E}$ ), or a 2-tailed Student's $t$ test (C). (H) Ten-week-old Ucp1-GFP mice were treated with dox-containing diet for 4 days before tissue harvest. PACT-cleared BAT from Ucp1-GFP mice and immunolabeled with GFP (green) and sympathetic neuron marker tyrosine hydroxylase (TH) (purple) antibody. Scale bar: $30 \mu \mathrm{m}$. Images are representative of 3 independent experiments.

seq. In the AdipoChaser-YFP mice (Figure 4A), $\mathrm{YFP}^{+}$cells (Adipoq high-expressing brown adipocytes) primarily overlapped with Elovl6 (Figure 4B) as well as SDHA and COX IV (Figure 4, C and D), whereas large numbers of $\mathrm{YFP}^{+}$brown adipocytes did not overlap with FABP4 (Figure 4E) or PPAR $\gamma$ (Figure 4F). Thus, the protein levels of these important genes match with the expression pattern demonstrated by the scRNA-seq results.

Isolation of the 2 brown adipocyte subpopulations reveals their distinct metabolic statuses. To test whether the 2 brown adipocyte subpopulations have different mitochondrial respiratory capacity, we separately collected the 2 freshly isolated brown adipocyte subpopulations from BAT through mild centrifugation. With the Ucp1-GFP mice, a tet-responsive labeling system under the control of the $U c p 1$ promoter (Figure $5 \mathrm{~A}$ ), isolation and separation were verified based on the GFP signal intensity (Figure 5B). We first measured mitochondrial function through a Mito Stress Test Kit (Figure 5C). The BA-H, BA-L, white adipocytes, and primary BAT stromal vascular fraction (SVF) showed distinct levels of oxygen consumption as judged by the oxygen consumption rate (OCR). As the ATP synthesis rate is relatively low in brown adipocytes due to uncoupled respiration, it is not surprising that oligomycin (ATP synthase inhibitor) did not markedly alter OCR in brown adipocytes, whereas oligomycin decreased OCR by $59 \%$ in SVF and by $20 \%$ in white adipocytes (Figure 5 C). The basal respiration in the BA-H population was around 2-fold higher compared with the BA-L population. Both brown adipocyte subpopulations had substantially higher basal respiration compared with white adipocytes and the SVF (Figure 5D). Interestingly, the BA-H population had a maximal respiration rate very close to the BA-L population, indicating these brown adipocytes have high mitochondrial potential and are readily recruitable (Figure $5 \mathrm{E}$ ). The BA-H population isolated through mild centrifugation contains a low percentage of SVF. To obtain a purer BA-H population, we isolated the BA-H population from AdipoChaser-mT/mG mice through a magnetic bead-based method, taking advantage of the membrane-bound GFP. The OCR levels of both BA-L and BA-H subpopulations obtained through this alternative method were much lower compared with the cells obtained through mild centrifugation, which may be due to the much longer processing time involved for the isolation (Figure $5 \mathrm{~F}$ ). However, the difference in basal OCR is consistent between the 2 methods (Figure 5, C and F). Moreover, both BA-L and BA-H populations showed responses to norepinephrine, but the BA-H population had a more robust increase in the OCR (Figure 5F). We also measured the fatty acid uptake rate in the 2 brown adipocyte subpopulations, and consistent with the high Fabp4 mRNA and protein level (Figure 3F and Figure 4E), the BA-L population displayed much higher rates of fatty acid uptake (Figure $5 \mathrm{G}$ ). This result is consistent with a recent report that demonstrated that the uptake of nutrients by adjacent murine brown adipocytes is variable (36). Overall, these results demonstrate that the 2 brown adipocyte subpopulations have fundamentally distinct function and metabolic profiles.

Sympathetic innervation is not correlated with the distribution of 2 brown adipocyte subpopulations. We performed 3D profiling of BAT from the Ucp1-GFP mice housed at $24^{\circ} \mathrm{C}$. $\mathrm{UCP}^{+}\left(\mathrm{GFP}^{+}\right)$ brown adipocytes distributed in a patchy pattern (Figure $5 \mathrm{H}$ and Supplemental Video 1), confirming the scRNA-seq result that $U c p 1$ is also distinctly expressed in different subpopulations of brown adipocytes. The thermogenesis of brown adipocytes is governed by sympathetic innervation (37). The 3D architecture showed that compared with the less innervated white adipose tissues $(38,39)$, almost every brown adipocyte is heavily innervated with sympathetic neurons (Figure 5H and Supplemental Video 1). Thus, the diversity in thermogenic activity observed in these 2 brown adipocyte subpopulations is not determined by sympathetic innervation. Notably, the expression level of $\beta 3$-adrenergic receptor Adrb3 was enriched in the BA-H subpopulation (Supplemental Figure 7G). Therefore, the diverse thermogenic activity may be determined by the difference in the responsiveness of brown adipocytes to $\beta 3$-adrenergic signals.

Developmental timing of the 2 brown adipocyte subpopulations. Does BAT emerge developmentally as 2 distinct subpopulations? We looked into the Adipoq expression in brown adipocytes during development, by exposing AdipoChaser-LacZ mice to dox diet at various embryonic and postnatal stages (Figure 6A). When we exposed mice to dox diet during E3-E10, very few (less than 1\%) brown adipocytes were labeled as LacZ ${ }^{+}$cells upon examining the tissue at 4 weeks of age (Figure 6B). When mice were exposed to dox diet during E7-E14, brown adipocytes showed a heterogeneous pattern of LacZ ${ }^{+}$cells, with some regions carrying more than $92 \%$ and other regions displaying less than $10 \% \mathrm{LacZ}^{+}$signal when examined at 4 weeks of age (Figure $6 \mathrm{C}$ ). When mice were exposed to dox diet during E9-E16, brown adipocytes showed uniform positive labeling of $\mathrm{LacZ}^{+}$cells when examined at 4 weeks of age (Figure 6D). These observations indicate that brown adipocyte differentiation is initiated as early as E10, and that all brown 


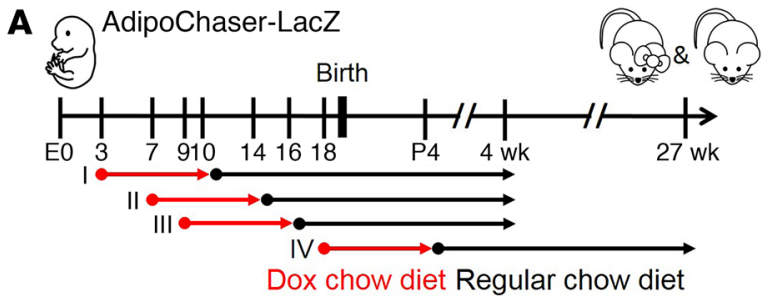

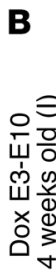
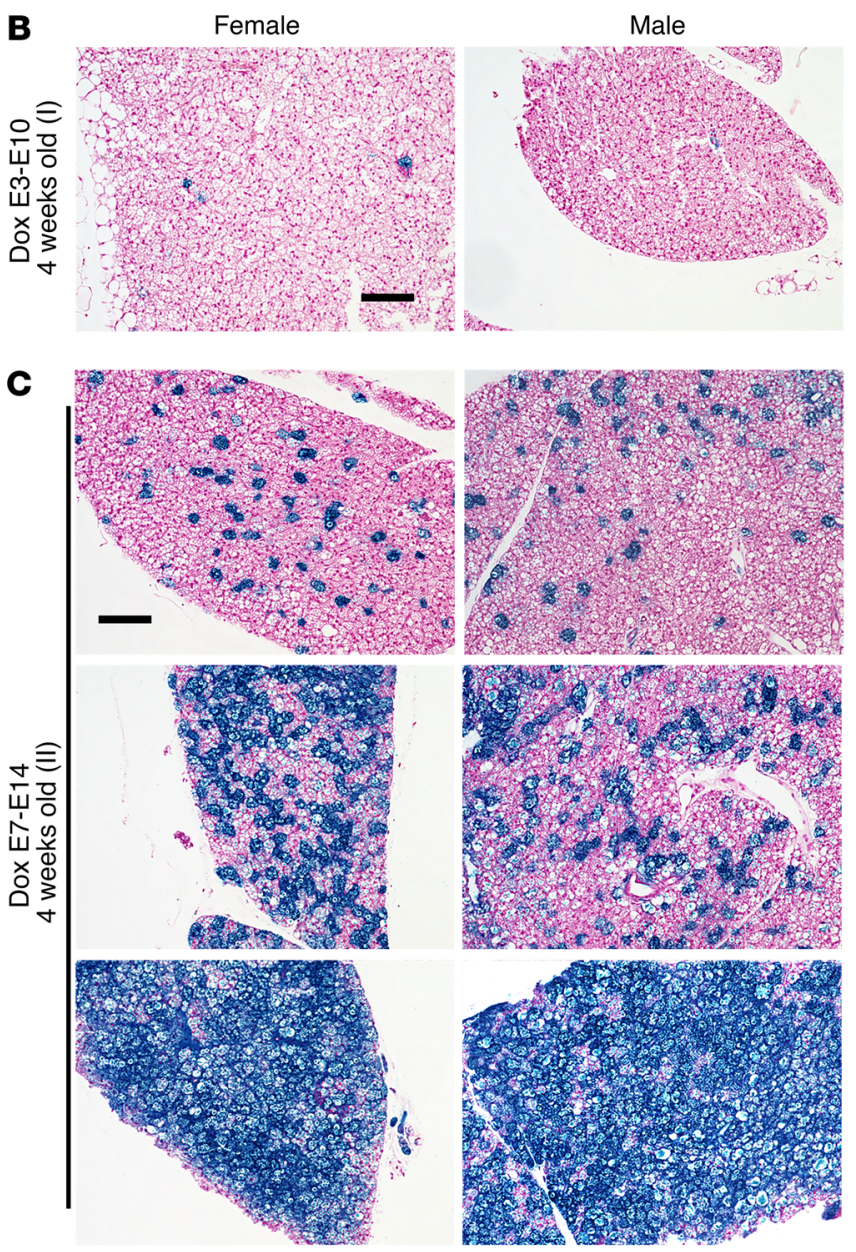

G

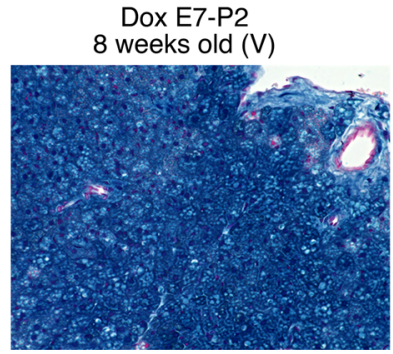

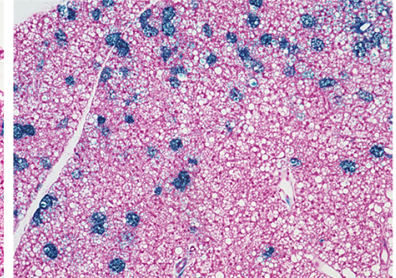

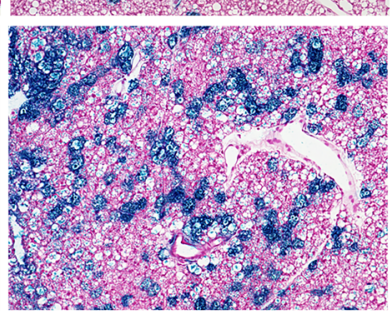

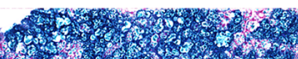

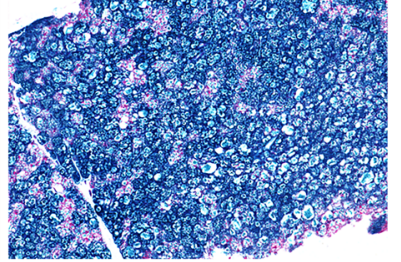

Dox P3-P10 8 weeks old (VI)

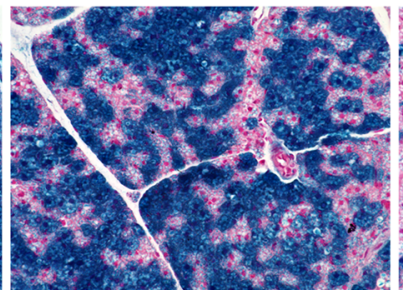

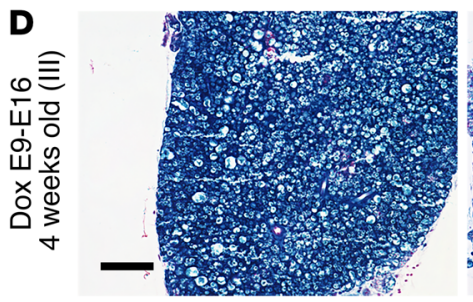
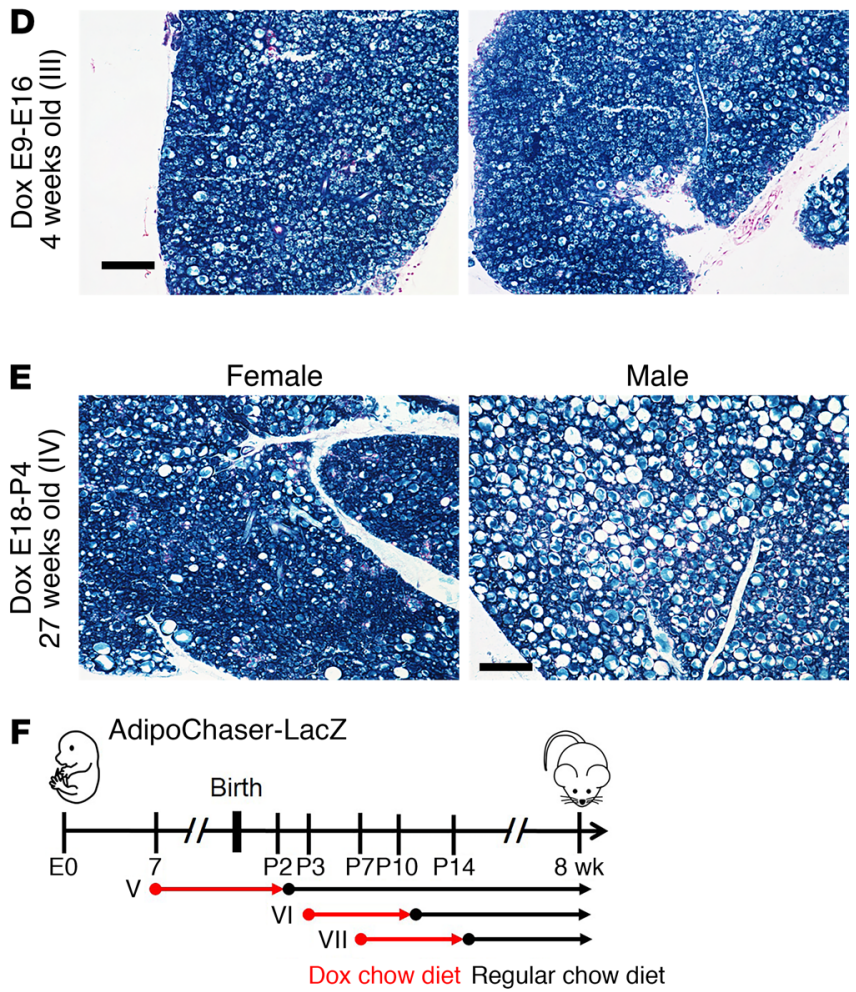

Figure 6. Brown adipocytes were born as adiponectin high expressors, and heterogeneity establishes after birth. (A) Female mice carrying only Adn-rtTA and Rosa26-loxP-stop-loxP-LacZ were bred with AdipoChaser-LacZ male mice. When these female mice were pregnant, they were exposed to dox-

containing chow diet during E3-E10 (I), E7-E14 (II), E9-E16 (III), or E18- P4(IV), and kept on regular chow diet thereafter. Offspring of the female mice were genotyped, and AdipoChaser-LacZ mice of both sexual phenotypes were used for LacZ staining when they were 4 weeks or 27 weeks old. (B-D) Representative X-gal staining of BAT from 4-week-old AdipoChaser-LacZ mice that were on dox diet for the indicated number of days during development. (E) Representative X-gal staining of BAT from 27-week-old AdipoChaser-LacZ mice that were on dox diet during E18-P4. Scale bars: $100 \mu \mathrm{m}$ (B-E). (F) Female mice carrying only Adn-rtTA and Rosa26-loxP-stop-loxP-LacZ were bred with AdipoChaser-LacZ male mice. When the female mice were pregnant, they were exposed to dox-containing chow diet during E7-P2 (V), P3-P10 (VI), or P7-P14 (VII), and kept on regular chow diet thereafter. Offspring of the female mice were genotyped, and male AdipoChaser-LacZ mice were used for LacZ staining when they were 8 weeks old. (G) Representative X-gal staining of BAT from the AdipoChaser-LacZ mice that were on dox diet for the indicated number of days during development. Scale bar: $50 \mu \mathrm{m}$. All images are representative of 3 independent experiments. 

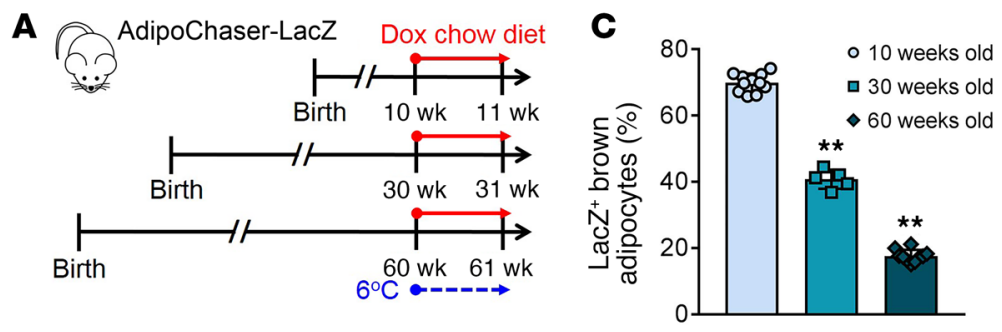

B
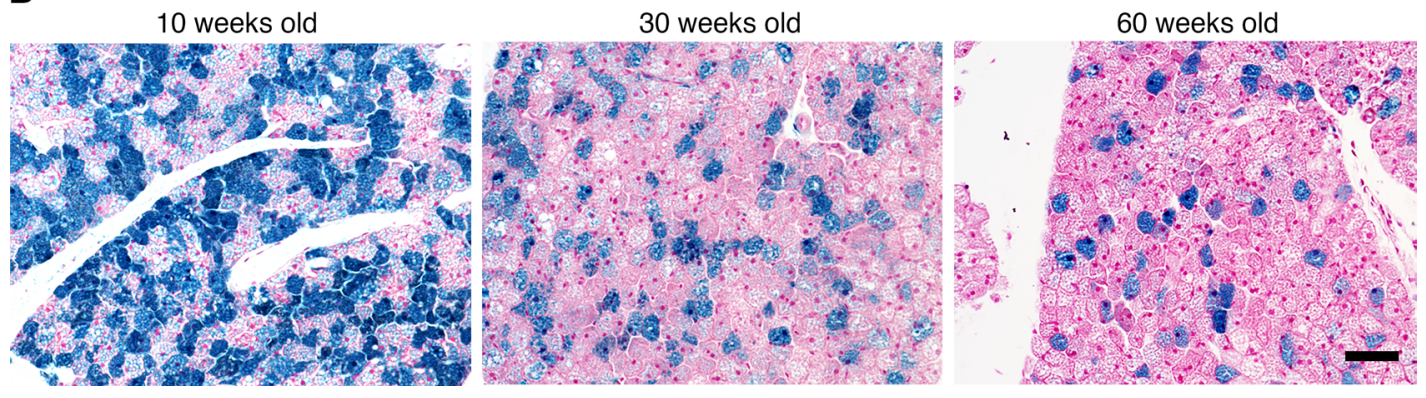

Figure 7. The recruitment of BA-H during cold exposure declines with age. (A) AdipoChaser-LacZ male mice were kept on normal chow until 30 or 60 weeks of age. Mice were then exposed to $6^{\circ} \mathrm{C}$ for 7 days while treated with dox-containing chow diet. (B) Representative X-gal staining of BAT from mice of different age exposed to $6^{\circ} \mathrm{C}$. Scale bar: $50 \mu \mathrm{m}$. (C) Quantification of the percentage of Lac $Z^{+}$brown adipocytes in the total brown adipocytes. $n=11$ mice (10 weeks old); $n=5$ mice (30 weeks old); $n=10$ mice (60 weeks old). ${ }^{* *} P<0.01$. Statistical significance was assessed using a 1 -way ANOVA followed by Tukey's multiple comparisons test. All images are representative of 3 independent experiments.

adipocytes have initiated differentiation and started to express Adipoq by E16. Thus, adiponectin can be used as a terminal differentiation marker for both brown adipocytes and white adipocytes (19). At the age of 27 weeks, for the mice that were exposed to dox diet during E18-P4, the brown adipocytes continued to display a uniformly positive LacZ labeling (Figure 6E), indicating that the turnover rate for brown adipocytes is extremely low in the adult stage at room temperature. Thus, the Adipoq low-expressing brown adipocytes are not newly generated after birth.

We subsequently narrowed down the time frame during which the BAT develops heterogeneity through the interconversion (Figure 6F). When AdipoChaser-LacZ mice were exposed to dox diet during E7-P2, their brown adipocytes showed a uniform $\mathrm{LacZ}^{+}$labeling when examined at 8 weeks of age (adult stage) (Figure 6G). When mice were exposed to dox diet during P3-P10 or P7-P14, $56 \%$ or $42 \%$ of their brown adipocytes were labeled as LacZ ${ }^{+}$cells, which is close to the percentage at the adult stage (38\% in Figure 1B). These experiments indicate that the BAT transcriptional program becomes heterogeneous shortly after birth, and the ratio of Adipoq high-expressing and low-expressing brown adipocytes becomes stable around P7.

The interconversion of $B A-L$ to $B A-H$ declines with age, but not HFD feeding. It has been suggested that decreased BAT thermogenesis is associated with the accumulation of body fat, as well as age (40-42). Therefore, we checked if high fat diet-induced (HFD-induced) obesity reduces the interconversion of Adipoq low expressers to high expressers during cold exposure (Supplemental Figure 8, A and B). When AdipoChaser-LacZ mice were housed at $6^{\circ} \mathrm{C}$, the percentages of $\mathrm{LacZ}^{+}$brown adipocytes from HFD-fed mice (47\%) were comparable to brown adipocytes from chow-fed mice (45\%) (Supplemental Figure 8, C and D). At $24^{\circ} \mathrm{C}$, HFD-fed mice had even higher percentages of $\mathrm{LacZ}^{+}$brown adipocytes
(23\% vs. $12 \%)$. Thus, HFD feeding does not impair the recruitment of Adipoq high-expressing brown adipocytes during cold exposure. However, even in the chow-fed group, these 21-week-old mice had a markedly lower percentage of $\mathrm{LacZ}^{+}$brown adipocytes at both $6^{\circ} \mathrm{C}$ and $24^{\circ} \mathrm{C}$ compared with 8-week-old mice (Figure 1, A and $\mathrm{B})$, indicating that there is a decline in the number of Adipoq high expressers with age. When older mice were housed at $6^{\circ} \mathrm{C}$ (Figure $7 \mathrm{~A})$, the percentage of $\mathrm{LacZ}^{+}$brown adipocytes further dropped to below 40\% (30-week-old) and 20\% (60-week-old) (Figure 7, $\mathrm{B}$ and $\mathrm{C})$. These results indicate that the ability of BAT to recruit Adipoq high-expressing brown adipocytes during cold exposure is substantially reduced with age.

\section{Discussion}

We report the discovery of a low-thermogenic brown adipocyte subpopulation with unique molecular and metabolic features, coexisting with the classical brown adipocytes in vivo. The results presented here offer critical insight toward our understanding of how brown adipose tissue thermogenesis is regulated at the cellular level. The discovery of the new low-thermogenic subpopulation is of great interest since this population of cells does not have typical brown adipocyte morphology and displays a unique metabolic profile. However, the exact function of this subpopulation is largely unknown. These brown adipocytes have relatively large lipid droplets and low mitochondrial content and an extremely low respiration rate, compared with the high-thermogenic subpopulation. Are these brown adipocytes in a resting status and readily recruitable to convert into high-thermogenic cells? Or do they have critical metabolic functions other than thermogenesis? As the low-thermogenic brown adipocytes have a much higher rate of fatty acid intake, these cells may have an indispensable role for the functional integrity of the thermogenic activity of the whole 
BAT. The high-thermogenic subpopulation represents the extensively studied classical brown adipocyte subtype, which has the potential ability to further increase Ucp1 expression and thermogenesis upon cold stimulation. It is noteworthy that most of the human studies detect BAT based on glucose uptake, as BAT exhibits high uptake of fluorine-18 fludeoxyglucose on positron emission tomography (PET). This detection method may miss the lower thermogenic brown adipocytes, which have high fatty acid uptake rate.

Adiponectin is considered a white adipocyte marker since it is more abundantly expressed in the white adipocyte. However, it is not surprising to observe a higher Adipoq expression in the high-thermogenic brown adipocytes, as adiponectin positively regulates mitochondrial biogenesis and activity (43-45). Recent $3 \mathrm{D}$ adipose tissue imaging reveals that cold-induced generation of beige adipocytes in the subcutaneous adipose tissue depends on the density of sympathetic innervation $(38,39)$. Here, we show that sympathetic innervation in BAT is much denser than that in white adipose tissue. Thus, the thermogenic heterogeneity of brown adipocytes is not correlated to sympathetic innervation. However, it is still possible that norepinephrine is differentially secreted by each sympathetic neuron. Notably, the expression level of $\beta 3$-adrenergic receptor Adrb3 was enriched in the BA-H subpopulation. Therefore, the diverse thermogenic activity may be determined by the difference in the responsiveness of brown adipocyte to $\beta 3$-adrenergic signals.

Developmentally, as newborn pups require much higher thermogenic activity, it is not surprising that all brown adipocytes are born as Adipoq high expressers and potentially have high thermogenic activity. Interestingly, a subpopulation of brown adipocytes gradually converts into Adipoq low expressers after birth. The establishment of heterogeneity after birth is likely due to the heterogeneous lineages of brown adipocyte precursors during development. However, it is also possible that the 2 brown adipocyte subpopulations are not born to be different, and they may undergo a switching mechanism even at room temperature, taking dynamic turns to function as high-thermogenic cells. As the interscapular BAT is the first adipose depot to develop in the mouse, BAT may serve as the primary site for adiponectin expression and secretion in these very early stages of life. When white adipose depots development initiates later in life, these tissues then take over as the primary sites for adiponectin production. When mice in the adult stage are exposed to cold, other than a conversion of BA-L into BA-H population, BAT may also undergo de novo adipogenesis, especially when mice are exposed to the cold for a long period of time $(46,47)$. Importantly, the conversion of low-thermogenic brown adipocytes into high-thermogenic adipocytes upon cold exposure is impaired with old age, but not by HFD feeding. This may offer a new explanation for the age-associated decline in brown adipose tissue thermogenic activity.

Future studies will need to address the metabolic functions and lineages of the low-thermogenic brown adipocyte subpopulation, as well as the molecular mechanisms that regulate the interconversion between the 2 subpopulations. More importantly, it will be interesting to determine why the number of high-thermogenic brown adipocytes declines with age. Unmasking the complex physiology of BAT thermogenesis is essential to improve our ability to identify effective therapeutic approaches for metabolic disorders. Future strategies that promote the low-thermogenic brown adipocytes to convert into a population of high-thermogenic cells may greatly enhance brown adipose tissue thermogenesis, which may have potential for the treatment of obesity and diabetes.

\section{Methods}

Detailed methods are in the Supplemental Material.

The scRNA-seq data have been deposited in NCBI Gene Expression Omnibus database (accession number GSE125269).

Statistics. The results are mean \pm SD. Differences were analyzed by various methods as indicated in figure legends. All measurements were taken from individual samples.

Study approval. The City of Hope IACUC approved all animal experiments.

\section{Author contributions}

QAW, PES, and AS designed the experiments. QAW, PES, and LJ wrote the manuscript. AS and QAW handled all the mouse experiments and performed $\beta$-gal staining. AL performed histological sectioning. AS performed the mitochondrial membrane potential test and immunofluorescence staining. AS prepared primary brown adipocytes and XW conducted and analyzed scRNA-seq experiments. AS, QAW, LM, TN and WD performed the seahorse and fatty acid intake experiment. AS, MJJ, HZ, and BA performed BAT tissue clearing and 3D imaging. AS, ZL, and MMM performed the transmission electron microscopy. MS, JT, JMH, YL, YZ, LJ, and VG contributed to experimental design and discussion. All authors approved the final manuscript.

\section{Acknowledgments}

The authors are grateful to Jiandie Lin, Li Ye, and members of the Diabetes and Metabolism Research Institute for discussions and comments. The authors thank the City of Hope Animal Resource Center, Integrative Genomics Core, Electron Microscopy and Atomic Force Microscopy Core, Light Microscopy Core, Pathology (Solid Tumor) Core (supported by NIH P30CA033572), Analytical Cytometry Core, and City of Hope Comprehensive Cancer Center for guidance and assistance for experiments. This study was supported by NIH grants K01DK107788, R03HD095414, and R56AG063854 (to QAW) and R01DK55758, R01DK099110, P01DK088761, and P01AG051459 (to PES). QAW was also supported by City of Hope Caltech-COH Initiative Award and the American Diabetes Association Junior Faculty Development Award (1-19-JDF-023). PES was also supported by an unrestricted research grant from the Novo Nordisk Foundation and by a grant from the Kristian Gerhard Jebsen Foundation. This work was also supported by the Beckman Institute for CLARITY, Optogenetics and Vector Engineering Research for technology development and broad dissemination (http://clover.caltech.edu/) (to VG) and Caltech Divisional Postdoctoral Fellowship (to MJJ).

Address correspondence to: Qiong (Annabel) Wang, Department of Molecular \& Cellular Endocrinology, City of Hope, 1500 East Duarte Road, Duarte, California 91010, USA. Phone: 626.218.6419; Email: qwang@coh.org. 
1. Orava J, et al. Different metabolic responses of human brown adipose tissue to activation by cold and insulin. Cell Metab. 2011;14(2):272-279.

2. Ouellet V, et al. Brown adipose tissue oxidative metabolism contributes to energy expenditure during acute cold exposure in humans. JClin Invest. 2012;122(2):545-552.

3. Chondronikola M, et al. Brown adipose tissue improves whole-body glucose homeostasis and insulin sensitivity in humans. Diabetes. 2014;63(12):4089-4099.

4. Bartelt A, et al. Brown adipose tissue activity controls triglyceride clearance. Nat Med. 2011;17(2):200-205.

5. Stanford KI, et al. Brown adipose tissue regulates glucose homeostasis and insulin sensitivity. J Clin Invest. 2013;123(1):215-223.

6. Chouchani ET, Kazak L, Spiegelman BM. New advances in adaptive thermogenesis: UCP1 and beyond. Cell Metab. 2019;29(1):27-37.

7. Lowell BB, et al. Development of obesity in transgenic mice after genetic ablation of brown adipose tissue. Nature. 1993;366(6457):740-742.

8. Kopecky J, Clarke G, Enerbäck S, Spiegelman B, Kozak LP. Expression of the mitochondrial uncoupling protein gene from the aP2 gene promoter prevents genetic obesity. J Clin Invest. 1995;96(6):2914-2923.

9. Crane JD, et al. Inhibiting peripheral serotonin synthesis reduces obesity and metabolic dysfunction by promoting brown adipose tissue thermogenesis. Nat Med. 2015;21(2):166-172.

10. Gnad T, et al. Adenosine activates brown adipose tissue and recruits beige adipocytes via A2A receptors. Nature. 2014;516(7531):395-399.

11. Bertholet AM, et al. Mitochondrial patch clamp of beige adipocytes reveals UCP1-positive and UCP1-negative cells both exhibiting futile creatine cycling. Cell Metab. 2017;25(4):811-822.e4.

12. Lee KY, et al. Tbx15 defines a glycolytic subpopulation and white adipocyte heterogeneity. Diabetes. 2017;66(11):2822-2829.

13. Lee KY, Luong Q, Sharma R, Dreyfuss JM, Ussar S, Kahn CR. Developmental and functional heterogeneity of white adipocytes within a single fat depot. $E M B O$ J. 2019;38(3):e99291.

14. Chen Y, et al. Thermal stress induces glycolytic beige fat formation via a myogenic state. Nature. 2019;565(7738):180-185.

15. Cinti S, et al. CL316,243 and cold stress induce heterogeneous expression of UCP1 mRNA and protein in rodent brown adipocytes. J Histochem Cytochem. 2002;50(1):21-31.

16. Spaethling JM, et al. Single-cell transcriptomics and functional target validation of brown adipocytes show their complex roles in metabolic homeostasis. FASEB J. 2016;30(1):81-92.

17. Wikstrom JD, et al. Hormone-induced mitochondrial fission is utilized by brown adipocytes as an amplification pathway for energy expenditure. EMBO J. 2014;33(5):418-436.

18. Xie TR, Liu CF, Kang JS. Sympathetic transmitters control thermogenic efficacy of brown adipocytes by modulating mitochondrial complex V. Signal Transduct Target Ther. 2017;2:17060.

19. Wang QA, Tao C, Gupta RK, Scherer PE. Tracking adipogenesis during white adipose tissue development, expansion and regeneration. Nat Med. 2013;19(10):1338-1344.

20. Wang QA, et al. Reversible de-differentiation of mature white adipocytes into preadipocyte-like precursors during lactation. Cell Metab. 2018;28(2):282-288.e3.

21. Childs BG, Baker DJ, Wijshake T, Conover CA, Campisi J, van Deursen JM. Senescent intimal foam cells are deleterious at all stages of atherosclerosis. Science. 2016;354(6311):472-477.

22. Baker DJ, et al. Naturally occurring p16(Ink4a)-positive cells shorten healthy lifespan. Nature. 2016;530(7589):184-189.

23. Ye R, et al. Impact of tamoxifen on adipocyte lineage tracing: Inducer of adipogenesis and prolonged nuclear translocation of Cre recombinase. Mol Metab. 2015;4(11):771-778.

24. Harms M, Seale P. Brown and beige fat: development, function and therapeutic potential. Nat Med. 2013;19(10):1252-1263.

25. Chouchani ET, et al. Mitochondrial ROS regulate thermogenic energy expenditure and sulfenylation of UCP1. Nature. 2016;532(7597):112-116.

26. Mills EL, et al. Accumulation of succinate controls activation of adipose tissue thermogenesis. Nature. 2018;560(7716):102-106.

27. Crewe C, et al. An endothelial-to-adipocyte extracellular vesicle axis governed by metabolic state. Cell. 2018;175(3):695-708.e13.

28. Kazak L, et al. A creatine-driven substrate cycle enhances energy expenditure and thermogenesis in beige fat. Cell. 2015;163(3):643-655.

29. Tontonoz P, Hu E, Spiegelman BM. Stimulation of adipogenesis in fibroblasts by PPAR gamma 2, a lipid-activated transcription factor. Cell. 1994;79(7):1147-1156.

30. Hu E, Tontonoz P, Spiegelman BM. Transdifferentiation of myoblasts by the adipogenic transcription factors PPAR gamma and C/EBP alpha. Proc Natl Acad Sci USA. 1995;92(21):9856-9860.

31. Wang QA, et al. Distinct regulatory mechanisms governing embryonic versus adult adipocyte maturation. Nat Cell Biol. 2015;17(9):1099-1111.

32. Inagaki T, Sakai J, Kajimura S. Transcriptional and epigenetic control of brown and beige adipose cell fate and function. Nat Rev Mol Cell Biol. 2016;17(8):480-495

33. Lin J, et al. Transcriptional co-activator PGC-1 alpha drives the formation of slow-twitch muscle fibres. Nature. 2002;418(6899):797-801.

34. Lin J, et al. PGC-1beta in the regulation of hepatic glucose and energy metabolism. J Biol Chem. 2003;278(33):30843-30848.

35. Rajakumari S, et al. EBF2 determines and maintains brown adipocyte identity. Cell Metab. 2013;17(4):562-574.

36. He C, et al. NanoSIMS imaging reveals unexpected heterogeneity in nutrient uptake by brown adipocytes. Biochem Biophys Res Commun. 2018;504(4):899-902.

37. Morrison SF, Madden CJ, Tupone D. Central neural regulation of brown adipose tissue thermogenesis and energy expenditure. Cell Metab. 2014;19(5):741-756

38. Jiang H, Ding X, Cao Y, Wang H, Zeng W. Dense intra-adipose sympathetic arborizations are essential for cold-induced beiging of mouse white adipose tissue. Cell Metab. 2017;26(4):686-692.e3.

39. Chi J, et al. Three-dimensional adipose tissue imaging reveals regional variation in beige fat biogenesis and PRDM16-dependent sympathetic neurite density. Cell Metab. 2018;27(1):226-236.e3

40. Jung RT, Shetty PS, James WP, Barrand MA, Callingham BA. Reduced thermogenesis in obesity. Nature. 1979;279(5711):322-323.

41. Yoneshiro T, et al. Age-related decrease in cold-activated brown adipose tissue and accumulation of body fat in healthy humans. Obesity (Silver Spring). 2011;19(9):1755-1760.

42. Tajima K, et al. Mitochondrial lipoylation integrates age-associated decline in brown fat thermogenesis. Nat Metab. 2019;1(9):886-898.

43. Iwabu M, et al. Adiponectin and AdipoR1 regulate PGC-1alpha and mitochondria by $\mathrm{Ca}(2+)$ and AMPK/SIRT1. Nature. 2010;464(7293):1313-1319.

44. Kusminski CM, et al. MitoNEET-driven alterations in adipocyte mitochondrial activity reveal a crucial adaptive process that preserves insulin sensitivity in obesity. Nat Med. 2012;18(10):1539-1549.

45. Qiao L, Kinney B, Yoo HS, Lee B, Schaack J, Shao J. Adiponectin increases skeletal muscle mitochondrial biogenesis by suppressing mitogenactivated protein kinase phosphatase-1. Diabetes. 2012;61(6):1463-1470.

46. Bukowiecki LJ, Géloën A, Collet AJ. Proliferation and differentiation of brown adipocytes from interstitial cells during cold acclimation. Am J Physiol.1986;250(6 Pt 1):C880-C887.

47. Lee YH, Petkova AP, Konkar AA, Granneman JG. Cellular origins of cold-induced brown adipocytes in adult mice. FASEB J. 2015;29(1):286-299. 